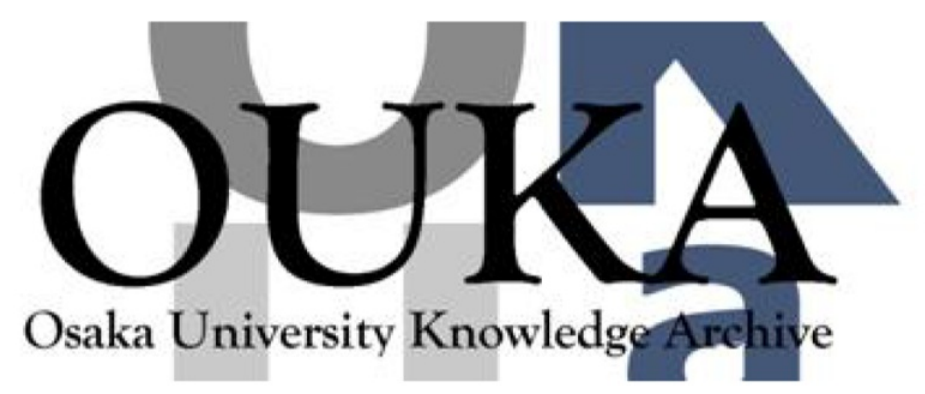

\begin{tabular}{|c|l|}
\hline Title & $\begin{array}{l}\text { Dispersion of resonant raman scattering in } \pi- \\
\text { conjugated polymers: Role of the even parity } \\
\text { excitons }\end{array}$ \\
\hline Author(s) & $\begin{array}{l}\text { Ozaki, M. ; Ehrenfreund, E. ; Benner, R. E. et } \\
\text { al. }\end{array}$ \\
\hline Citation & Physical Review Letters. 79(9) p. 1762-p. 1765 \\
\hline Issue Date & $1997-09-01$ \\
\hline oaire:version & VoR \\
\hline URL & https://hdl. handle. net/11094/75864 \\
\hline rights & $\begin{array}{l}\text { Copyright (1997) by the American Physical } \\
\text { Society }\end{array}$ \\
\hline Note & \\
\hline
\end{tabular}

Osaka University Knowledge Archive : OUKA

https://ir. Library. osaka-u. ac. jp/

Osaka University 


\title{
Dispersion of Resonant Raman Scattering in $\pi$-Conjugated Polymers: Role of the Even Parity Excitons
}

\author{
M. Ozaki, ${ }^{1,2}$ E. Ehrenfreund, ${ }^{3}$ R. E. Benner, ${ }^{1}$ T. J. Barton, ${ }^{4}$ K. Yoshino,${ }^{2}$ and Z. V. Vardeny ${ }^{1}$ \\ ${ }^{1}$ Departments of Physics and Electrical Engineering, University of Utah, Salt Lake City, Utah 84112 \\ ${ }^{2}$ Department of Electronic Engineering, Osaka University, Suita Osaka 565 Japan \\ ${ }^{3}$ Department of Physics and Solid State Institute, The Technion, Haifa 32000, Israel \\ ${ }^{4}$ Department of Chemistry, Iowa State University, Ames, Iowa 50011
}

(Received 2 January 1997)

\begin{abstract}
Resonant Raman scattering dispersion of the most strongly coupled phonon frequencies with the excitation laser photon energy is measured in terms of a dispersion rate parameter $D$ and quantified in a variety of $\pi$ conjugated polymer films. $D$ was found to be large in nonluminescent polymers and small in luminescent polymers. We show that $D$ is determined by the dependence of the even parity excitons $\left(A_{g}\right)$ on the polymer conjugation length, and this may serve as a useful spectroscopy for the $2 A_{g}$ exciton in nonluminescent polymers where it is otherwise optically inactive. [S0031-9007(97)03892-1]
\end{abstract}

PACS numbers: 78.30.Jw, 71.35.-y, 71.38.+i, 78.55.Kz

Resonant Raman scattering (RRS) dispersion of the phonon frequencies with the excitation laser photon energy $E_{L}$ in $\pi$-conjugated polymers has been widely used to study the films' inhomogeneity [1-8]. Because of disorder, inhomogeneous films have a wide distribution of optical energy gaps $E_{g}$ and associated vibrational frequencies $\omega_{i}$ of the most strongly coupled vibrations. At resonance $E_{L}=E_{g}$ and thus a particular $E_{g}$ and a coupled set of $\omega_{i}$ are selected by $E_{L}$, causing the resonant phonon frequencies $\omega_{i}^{L}$ to shift with $E_{L}$.

This RRS dispersion has been most thoroughly studied in trans-polyacetylene $\left[t-\left(\mathrm{CH}_{x}\right)\right]$ and polydiacetylene (PDA) [8], where the most strongly coupled vibrations were found to blueshift by as much as $\approx 100 \mathrm{~cm}^{-1}$ when $E_{L}$ was changed between 1.9 and $3.4 \mathrm{eV}$ [1-7]. The inhomogeneity in $E_{g}$ and $\omega_{i}$ was originally attributed to a conjugation length $(N)$ distribution where $E_{g}$ and $\omega_{i}$ depend on $N[1,2]$; later models, however, have focused on more microscopic approaches [3,6,7]. Among other polymers that have been studied, polythiophene [9] and poly ( $p$-phenylene vinylene) (PPV) [10] showed only a weak RRS dispersion, although a wide $E_{g}$ distribution could be inferred from their broad optical absorption and electroabsorption spectra [11].

At the same time, advances in understanding the excited electronic states in $\pi$-conjugated polymers have led researchers to recognize the importance of the excited state ordering. The photoluminescence (PL) quantum efficiency $\gamma$ and the resonant third-order nonlinear optical properties of these materials are determined by the energies and symmetries of a subset of the excited states, including a series of singlet excitons with odd $\left(n B_{u}\right)$ and even $\left(n A_{g}\right)$ parity lying below the continuum band threshold [12]. In particular, the relative energies of the lowest $B_{u}\left(1 B_{u}\right)$ and $A_{g}\left(2 A_{g}\right)$ excitons determined $\gamma$ [3]: If $E\left(2 A_{g}\right)<E\left(1 B_{u}\right)$, as in polymers with small dimerization $\delta$, then $\gamma$ is small because of the dipole forbidden character of the lowest singlet. Conversely, for
$E\left(2 A_{g}\right)>E\left(1 B_{u}\right)$, as in polymers with large dimerization $\delta, \gamma$ is large and these polymers might be considered as active materials for displays and laser-action applications.

In this Letter, we measure and quantify the RRS dispersion in a large group of $\pi$-conjugated polymers and show that it is inversely related to $\delta$ : The dispersion is most pronounced in nonluminescent polymers (NLP) with small $\delta$, whereas luminescent polymers (LP) with large $\delta$ show a much weaker dispersion. We establish that the RRS dispersion is determined by the dependences of $n A_{g}$ with $N$, rather than that of $1 B_{u}$ as thought before [17]. We further conjecture that in NLP the combination of small $\delta$ and $E\left(2 A_{g}\right)$, together with a large $E\left(2 A_{g}\right)$ dependence on $N$, leads to a strong RRS dispersion.

We report in this Letter RRS dispersion measured in a broad spectral range from 1.5 to $3.4 \mathrm{eV}$ in a variety of LP and NLP. As summarized in Table I, the LP studied consists of poly(alkylthiophene) (PAT-6), substituted PPV ( $s$-PPV), $s$-poly(phenylene-ethynylene) $(s$-PPE), and $s$-poly(phenylene-ethynylene phenylenevinylene) ( $s$-PPEPV), whereas the NLP contain $t-(\mathrm{CH})_{x}$, 4-butoxy carbonyl-methyluretane (4BCMU), substituted $t-(\mathrm{CH})_{x}\left[s-(\mathrm{CH})_{x}\right]$, poly(thienylene vinylene) (PTV) and two versions of poly(diethylene-silane) (PDES). We used free-standing films (5-10 $\mu \mathrm{m}$ thick) of these polymers, or films deposited on quartz substrates (1-2 $\mu$ m thick) by spin casting from chloroform solutions. Raman scattering data were obtained in a backscattering geometry using various excitation lasers. Scattered light was collected at $f / 1.2$ using an achromatic lens combination and was focused onto the entrance slit of a triple spectrograph (Spex 1877) equipped with 1200 grooves $/ \mathrm{mm}$ ruled grating. The Raman signal was recorded using an optical multichannel detection system with a liquid nitrogen cooled charge coupled device (photometrics).

As representatives of NLP we show in Figs. 1(a) and 1(b) RRS spectra of $s-(\mathrm{CH})_{x}$ and PDES, respectively, at various $E_{L}$ between 1.5 and $3.4 \mathrm{eV}$; the polymer repeat 
TABLE I. RRS dispersion rate $D$ and $E_{g}^{0}$ for luminescent and nonluminescent polymers.

\begin{tabular}{lcclcc}
\hline \hline & \multicolumn{3}{c}{ Luminescent polymers } & \multicolumn{3}{c}{ Nonluminescent polymers } \\
Material & $E_{g}^{0}(\mathrm{eV})$ & $D\left(10^{-3} \mathrm{eV}^{-1}\right)$ & \multicolumn{1}{c}{ Material } & $E_{g}^{0}(\mathrm{eV})$ & $D\left(10^{-3} \mathrm{eV}^{-1}\right)$ \\
\hline$s$-PPE & 2.5 & 5 & PTV & 1.9 & 45 \\
$s$-PPEPV & 2.3 & 10 & PDES-P & 1.9 & 53 \\
$s$-PPV & 2.15 & 15 & $s$ - $(\mathrm{CH})_{x}$ & 1.9 & 62 \\
PAT-6 & 1.95 & 20 & PDES-R & 1.75 & 67 \\
& & & $t$ - $(\mathrm{CH})_{x}$ & 1.4 & 193 \\
& & & PDA(BCMU) & 2.25 & 330 \\
\hline \hline
\end{tabular}

units are also shown in the insets. In order to emphasize the RRS dispersion, we focus in Fig. 1 on the two most strongly coupled vibrations. These are the $\mathrm{C}-\mathrm{C}$ stretching mode $\left[\sim 1200 \mathrm{~cm}^{-1}\right.$ in $s-(\mathrm{CH})_{x}$ and $1080 \mathrm{~cm}^{-1}$ in PDES, respectively] and the $\mathrm{C}=\mathrm{C}$ stretching mode $\left[1500 \mathrm{~cm}^{-1}\right.$ in $s-(\mathrm{CH})_{x}$ and $1450 \mathrm{~cm}^{-1}$ in PDES, respectively]. As $E_{L}$ increases, the two respective RRS lines in each polymer broaden and monotonously shift to higher frequencies.

The RRS spectra of the substituted PPV and PPE, two of the most strongly LP in the class of $\pi$-conjugated polymers, and their repeat units are shown in Fig. 2 in the same $E_{L}$ spectral range as in Fig. 1. The intense PL causes the RRS measurements to be more difficult in LP compared to NLP, especially for $E_{L}$ in the range 2.4$2.7 \mathrm{eV}$. For this $E_{L}$ range, the emission was collected for short times in order to avoid detector saturation, and

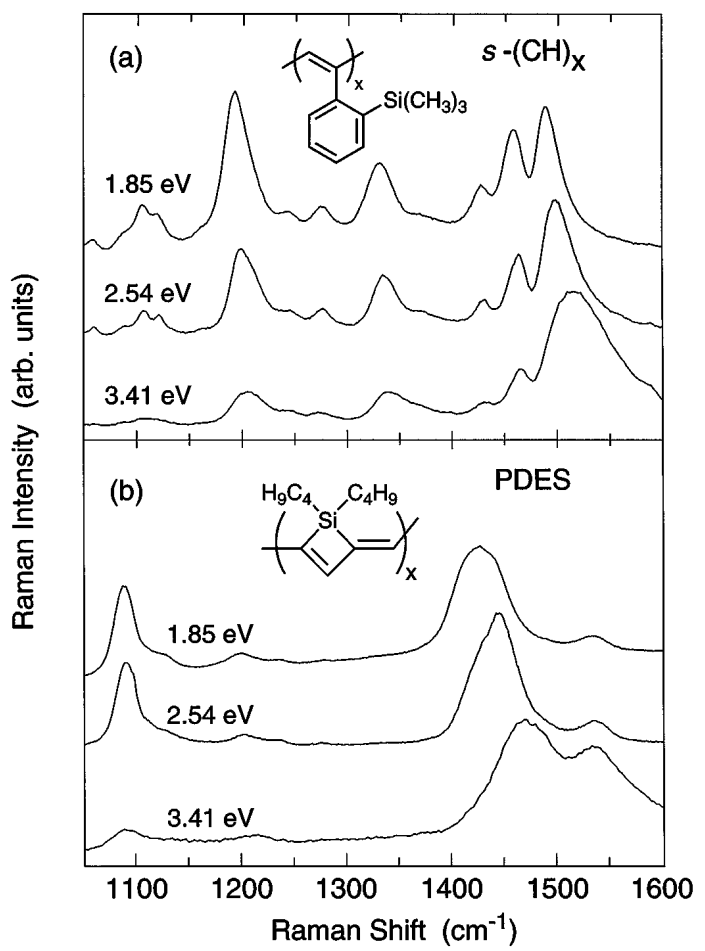

FIG. 1. Raman scattering spectra of (a) $s-(\mathrm{CH})_{x}$ and (b) PDES, which are nonluminescent polymers, at various laser photon energies. The polymer repeat units are given in the insets. then, as shown in Fig. 2(b) for PPE, the relatively broad PL background was numerically subtracted from the data to obtain the much weaker RRS spectrum. Surprisingly, in contrast to $s-(\mathrm{CH})_{x}$ and PDES (Fig. 1), almost no shift of the RRS frequencies $\omega_{i}^{L}$ with $E_{L}$ is observed in substituted PPV and PPE (Fig. 2), although their broad and structureless absorption and electroabsorption spectra indicate that substantial inhomogeneous distributions of $E_{g}$ [or $E\left(1 B_{u}\right)$ ] do exist in these films [11].

A unique way to quantify the RRS dispersion in $\pi$-conjugated polymers is by plotting the product of the square of the $m$ resonantly enhanced frequencies, $\prod_{i=1}^{m}\left(\omega_{i}^{L}\right)^{2}$, vs $E_{L}$ (the product rule relation) [3,7]. This relation accounts for the coupling among $\omega_{i}$, and thus is directly related to the RRS dispersion magnitude. In Figs. 3(a) and 3(b), we show the normalized product $\prod_{i=1}^{m}\left(\omega_{i}^{L} / \omega_{i}^{0}\right)^{2}$ vs $E_{L}$ for a variety of NLP and LP, respectively, where the normalization frequencies $\omega_{i}^{0}$ were determined as follows: For each polymer there is

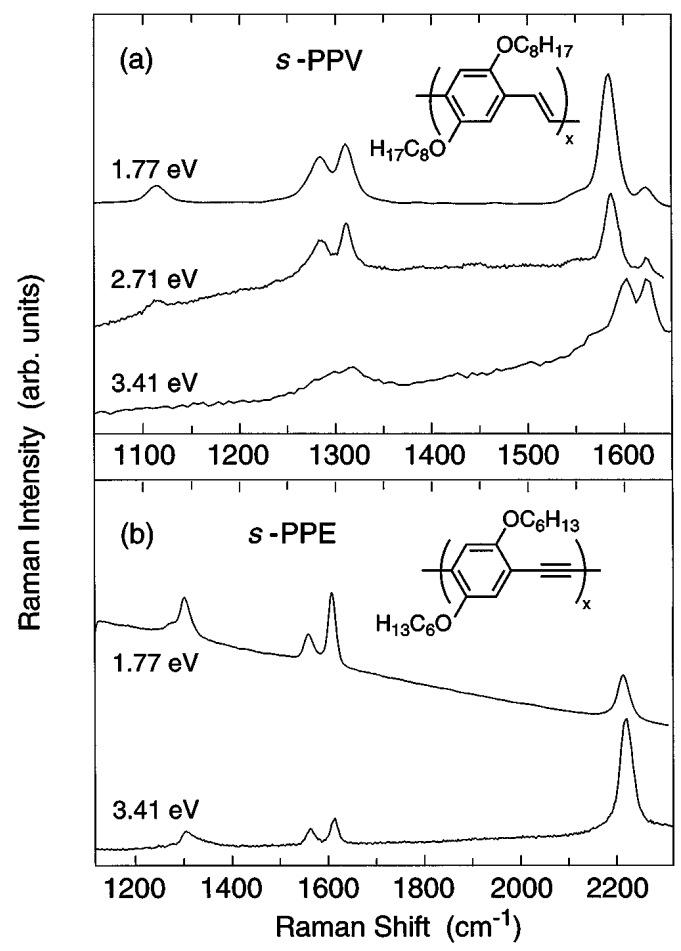

FIG. 2. Same as in Fig. 1 but for luminescent polymers: (a) $s$-PPV and (b) $s$-PPE. 

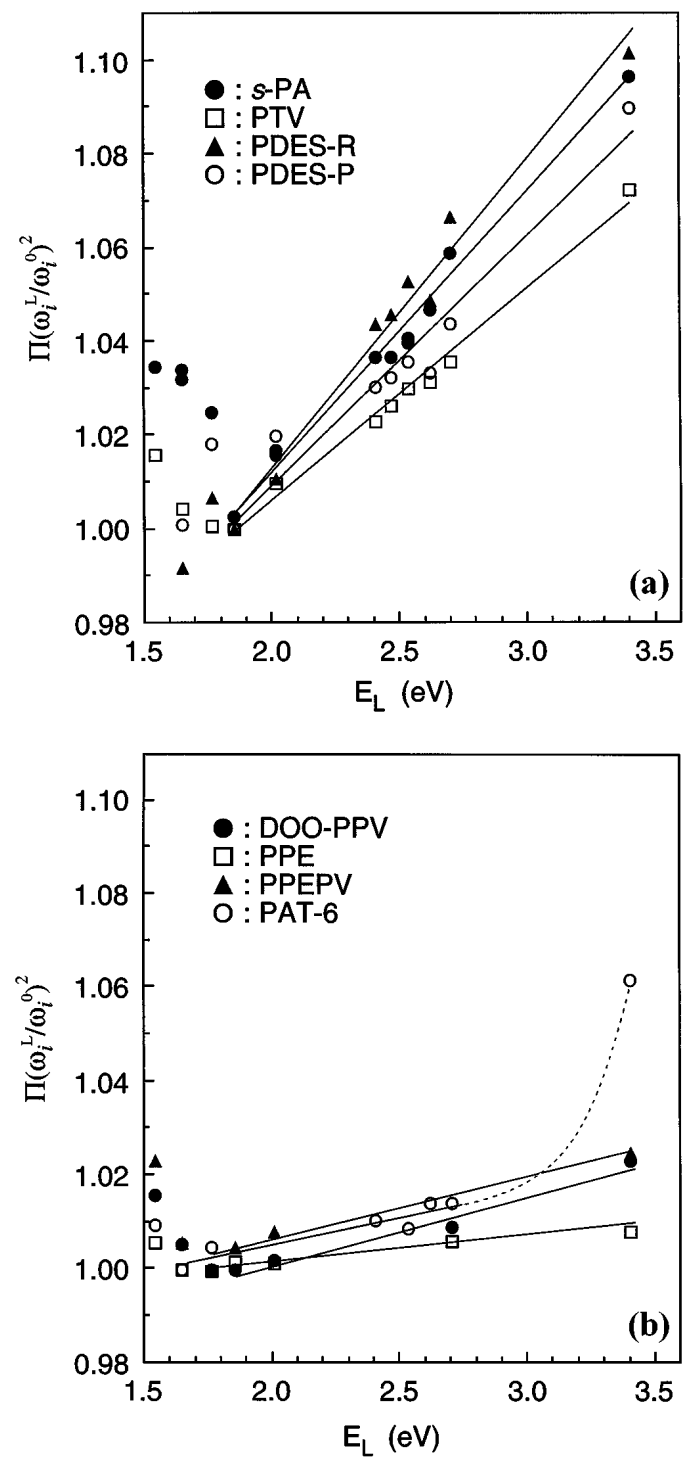

FIG. 3. The product rule relation $\prod_{i=1}^{m}\left(\omega_{i}^{L} / \omega_{i}^{0}\right)^{2}$ vs $E_{L}$ showing the RRS dispersion of various nonluminescent (a) and luminescent (b) polymers.

an $E_{L}$ value $E_{L}^{0}$ above which $\prod_{i=1}^{m}\left(\omega_{i}^{L} / \omega_{i}^{0}\right)^{2}$ linearly increases with $E_{L}$; for $E_{L}<E_{L}^{0}$ the product either does not change or slightly increases. This latter behavior is typical for nonresonance conditions, under which the entire frequency distribution can be observed [8]; resonance enhancement is evident only for $E_{L} \geq E_{L}^{0}$, and therefore we associate $E_{L}^{0}$ with $E_{g}$ of the longest chains in each sample, $E_{g}^{0}$. Accordingly, we denote the RRS frequencies measured at $E_{L}^{0}$ as $\omega_{i}^{0}$, and note that for each polymer these are the lowest RRS frequencies, which are associated with the longest respective polymer chains.

Since for $E_{L}>E_{L}^{0}, \prod_{i=1}^{m}\left(\omega_{i}^{L} / \omega_{i}^{0}\right)^{2}$ linearly depends on $E_{L}$ for both NLP and LP we quantify the RRS dispersion for each polymer by evaluating the dispersion rate parameter $D$ :

$$
D=d\left(\prod_{i=1}^{m}\left[\omega_{i}^{L} / \omega_{i}^{0}\right]^{2}\right) / d E_{L} .
$$

In Table I, we summarize the RRS dispersion rate $D$ for all measured polymers. Figures 3(a) and 3(b) and Table I show that $D$ is related to the excited state ordering as inferred from the PL efficiency. LP show little dispersion, whereas NLP show much stronger dispersion. Furthermore, as seen in Table I, among each polymer group, $D$ increases when $E_{g}^{0}$ decreases; PDA (4BCMU) is an exception to this empirical observation.

In the amplitude mode (AM) model [7] it is explicitly recognized that the strongest coupled vibrations are accompanied by a modulation in the $\pi$-electron energy [14]. For a multimode vibrational system, it has been shown that the product $\prod_{i=1}^{m} \omega_{i}^{2}$ is related to the generalized dimensionless AM force constant $\tilde{\lambda}$. Within this model, the unmodulated force constant, which is related to the $\sigma$ electron-phonon interaction, is renormalized by the $\pi$ electron interaction through $\tilde{\lambda}$. Thus $\tilde{\lambda}$ dependence on $E_{L}$ determines the RRS dispersion [7]. Soos and co-workers [15] have extended the AM model by including also quadratic electron-phonon couplings. In addition, they expressed $\tilde{\lambda}$ in terms of the $\pi$-electron susceptibility $\chi$ (where $\chi \sim 1-2 \tilde{\lambda}$ ), given by

$$
\chi=(8 / n) \sum_{n}\left|\left\langle n\left|\sum_{i}(-1)^{i} p_{i}\right| 0\right\rangle\right|^{2} / E_{n}=\sum_{n} a_{n} / E_{n} .
$$

In Eq. (2) $|0\rangle$ and $|n\rangle$ are the ground and excited $\pi$-electron states with energies 0 and $E_{n}$, respectively, and $p_{i}$ is the $\pi$-bond order operator [16] between sites $i$ and $i+1$. Since $p_{i}$ conserves parity [16] and $|0\rangle$ is even (i.e., $1 A_{g}$ ), it is obvious from Eq. (2) that $\chi$ is solely determined by the even parity electronic states $n A_{g}$. We thus conclude that $\chi$ (or $\tilde{\lambda}$ ) and, consequently, the RRS dispersion in conducting polymers do not depend on the properties and dispersion of the odd parity $n B_{u}$ states, and in particular not the $1 B_{u}\left(=E_{g}\right)$. It is therefore evident that the RRS dispersion results from the distribution of $E\left(n A_{g}\right)$ caused by the inhomogeneity in the films.

Assuming that the RRS dispersion arises from a distribution in $N$, we get through the relations $\prod\left(\omega_{i}\right)^{2} \sim \tilde{\lambda}$ and $\tilde{\lambda} \sim 1-\chi$, a modified product rule given by

$$
\prod_{i=1}^{m}\left(\frac{\omega_{i}(\nu)}{\omega_{i}^{0}}\right)^{2}=1+C\left(\chi_{0}-\chi_{\nu}\right)
$$

where $\nu \equiv 1 / N, \chi_{0} \equiv \chi$ for the smallest $\nu$ (longest $N$ ), and $C$ is a $\nu$ independent constant. Denoting $E\left(n A_{g}\right)$ by $E_{n}$, we write for $n \geq 2$ the dependence on $\nu[2,4]$ as $E_{n}(\nu)=E_{n}^{0}+\nu \beta_{n}$, where $\beta_{n}$ are $\nu$ independent constants and $E_{n}^{0}$ is $E_{n}$ at $\nu=0$. Similarly, we write for $E_{g}(\nu) \equiv E_{1 B_{u}}(\nu)=E_{g}^{0}+\nu \beta$. Under resonance conditions $E_{g}=E_{L}$ and, consequently, we find for the product [Eq. (3)]

$$
\prod_{i=1}^{m}\left[\omega_{i}^{L} / \omega_{i}^{0}\right]^{2}=1+D\left(E_{L}\right)\left(E_{L}-E_{g}^{0}\right),
$$


where the dispersion parameter $D\left(E_{L}\right)$ is given by

$$
D\left(E_{L}\right)=C \sum_{n} \frac{\beta_{n} a_{n}}{\beta\left(E_{n}^{0}\right)^{2}}\left[1+\frac{\beta_{n}\left(E_{L}-E_{g}^{0}\right)}{\beta E_{n}^{0}}\right]^{-1} .
$$

It is seen from Eq. (5) that for long chains where $\nu \ll$ $1, \beta_{n}\left(E_{L}-E_{g}^{0}\right) / \beta E_{n}^{0}=\beta_{n} \nu / E_{n}^{0} \ll 1$ and thus $D\left(E_{L}\right)$ becomes $E_{L}$ independent, given by the first part of (5):

$$
D=C \sum_{n} \frac{\beta_{n} a_{n}}{\beta\left(E_{n}^{0}\right)^{2}} .
$$

In this case, the product in Eq. (4) depends linearly on $E_{L}$, in agreement with the experiments (Fig. 3). Furthermore, it is seen from Eq. (6) that $D$ is dominated by the ratio $\beta_{2} / \beta$ and energy $E_{2}^{0} \equiv E^{0}\left(2 A_{g}\right)$ of the lowest lying $A_{g}$ exciton, namely, the $2 A_{g}$.

The excited state ordering in polymers and its dependence on $N$ have been extensively discussed in the literature [17-19]. It has been concluded that $E_{2}<E\left(1 B_{u}\right)$ (= $E_{g}$ ) for small dimetrization $\delta$, whereas the opposite order occurs for large $\delta[13,20]$. In addition, it was possible to calculate the dependencies of $E_{2}$ and $E_{g}$ on $N$, i.e., $\beta_{2}$ and $\beta$, respectively. It was found $[17,18]$ that $\beta_{2} / \beta \gg 1$ for NLP with small $\delta$, whereas $\beta_{2} / \beta \simeq 1$ for LP with large $\delta$. Since from our theoretical studies $D \approx\left(\beta_{2} / \beta\right) /\left[E_{2}\right]^{2}$ [Eq. (6)], we conclude that $D$ is large for NLP and small for LP in agreement with the experiments [Figs. 3(a) and 3(b) and Table I].

We can now understand in more detail the trend in $D$ among the polymers belonging to the two separate groups, as revealed in Table I. Since $D \sim\left(E_{2}\right)^{-2}$ and $E_{2}$ follows $E_{g}$ for polymers in the same group, $D$ should increase for polymers with smaller $E_{g}^{0}$, separately for LP and NLP. $\quad E_{g}^{0}$ as measured by the saturation of RRS dispersion is also given in Table I. Except for PDA, it is indeed seen that in each group, $D$ increases for polymers with smaller $E_{g}^{0}$ in agreement with our model. It is especially instructive to compare $D$ of $t-(\mathrm{CH})_{x}$ and $s-(\mathrm{CH})_{x}$ in the NLP group, since these polymers differ only by their side groups; their backbone structure is exactly the same (Fig. 1, inset). In this case we expect $D_{t} / D_{s} \simeq\left(E_{2, s} / E_{2, t}\right)^{2}$, where $t$ and $s$ refer to $t-(\mathrm{CH})_{x}$ and $s-(\mathrm{CH})_{x}$, respectively. $E_{2}$ was respectively measured in these two polymers by two photon absorption (TPA) [21] and transient strain spectroscopy [22], and found to be $E_{2, t}=1.1 \mathrm{eV}$ and $E_{2, s}=1.85 \mathrm{eV}$. Using these values in Eq. (6) we get $D_{t} / D_{s}=2.8$, whereas directly from RRS dispersion we measured $D_{t} / D_{s}=3.1$ (Table I).

The polymer PDA (4BCMU) is an exception among the NLP in Table I. Although it belongs to the group of polymers with large $D$, its $D$ is much larger than that expected from the above-mentioned trend in $E_{g}^{0}$. In another PDA polymer (PDA-PTS) it was found by TPA [23] that there are two $A_{g}$ states below $E_{g}(=2 \mathrm{eV})$, at $1.5 \mathrm{eV}$ and $1.8 \mathrm{eV}$, respectively. If we assume that similar to PTS, PDA-4BCMU also has two $A_{g}$ states below
$E_{g}$, then from Eq. (6) both states should substantially contribute to $D$, making it larger than expected from the $E_{g}^{0}$ trend (Table I).

In summary, we measured and quantified the RRS dispersion in the class of $\pi$-conjugated polymers in terms of a dispersion rate parameter $D$ and showed that $D$ is large for NLP and small for LP. Since the $\pi$-electron interaction renormalizes the RRS frequencies via an electronic susceptibility which contains only $A_{g}$ states, then $D$ is determined by the dependence of the $A_{g}$ states on the inhomogeneity in polymer conjugation length.

This work was supported in part by the DOE, Grants No. FG-03-96 and No. ER45490, and by the U.S-Israel Binational Science Foundation (BSF 94-256), Jerusalem, Israel. M. Ozaki acknowledges financial support from Murata Overseas Scholarship Foundation, Japan.

[1] H. Kuzmany, E. A. Imhoff, D. B. Fitchen, and A. Sarhangi, Phys. Rev. B 26, 7109 (1982).

[2] G. P. Brivio and E. Mulazzi, Chem. Phys. Lett. 95, 555 (1983).

[3] Z. Vardeny, E. Ehrenfreund, O. Brafman, and B. Horovitz, Phys. Rev. Lett. 51, 2326 (1983); 54, 75 (1985).

[4] H.E. Schaffer et al., J. Chem. Phys. 94, 4161 (1991).

[5] J. Kurti and H. Kuzmany, Phys. Rev. B 44, 597 (1991).

[6] M. Gussoni, C. Castiglioni, and G. Zerbi, in Spectroscopy of Advanced Materials, edited by R.J.H. Clark and R.E. Hester (John Wiley \& Sons, New York, 1991), p. 251.

[7] E. Ehrenfreund, Z. Vardeny, O. Brafman, and B. Horovitz, Phys. Rev. B 36, 1535 (1987).

[8] L.X. Zheng, R.E. Benner, Z.V. Vardeny, and G.L. Baker, Phys. Rev. B 42, 3235 (1990).

[9] Z. Vardeny et al., Synth. Met. 18, 183 (1987).

[10] Y. Furukawa, A. Sakamoto, and M. Tasumi, J. Phys. Chem. 93, 5354 (1989).

[11] M. Liess, S. Jeglinski, and Z.V. Vardeny (to be published)

[12] S. N. Dixit, D. Guo, and X. Mazumdar, Phys. Rev. B 43, 6781 (1991); S. Mazumdar and F. Guo, J. Chem. Phys. 100, 1665 (1994).

[13] Z. G. Soos, S. Etemad, D. S. Galvao, and S. Ramasesha, Chem. Phys. Lett. 194, 341 (1992).

[14] B. Horovitz, Solid State Commun. 41, 729 (1982).

[15] A. Girlando, A. Painelli, and Z. G. Soos, J. Chem. Phys. 98, 7459 (1993).

[16] C. A. Coulson, Proc. R. Soc. London A 169, 413 (1939).

[17] Z. G. Soos and R. Ramasesha, Phys. Rev. B 29, 5410 (1984).

[18] P. Tawan and K. Schulten, Phys. Rev. B 36, 4337 (1987).

[19] B.E. Kohler, C. Spangler, and C. Westerfield, J. Chem. Phys. 89, 5422 (1988).

[20] Z. G. Soos et al., Synth. Met. 54, 35 (1993).

[21] C. Halverson and A. J. Heeger, Chem. Phys. Lett. 216, 488 (1993).

[22] G. S. Kanner, S. Frolov, and Z. V. Vardeny, Mod. Phys. Lett. B 9, 1701 (1995).

[23] B. Lawrence et al., Phys. Rev. Lett. 73, 597 (1994). 